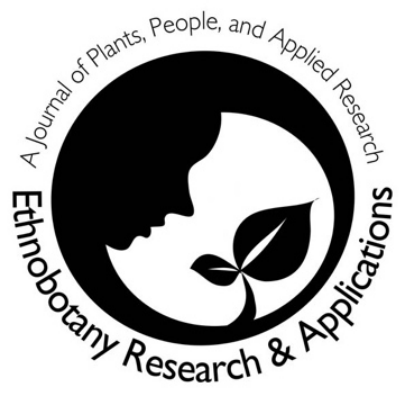

\title{
The ethnic diversities in animal-human interactions in former Jammu and Kashmir State- India
}

Musheerul Hassan, Shiekh Marifatul Haq, Umer Yaqoob, Saima Hamid, Muhammad Altaf and Rainer W. Bussmann

\section{Correspondence}

Musheerul Hassan ${ }^{1}$, Shiekh Marifatul Haq ${ }^{2}$, Umer Yaqoob ${ }^{2 \star}$, Saima Hamid ${ }^{3}$, Muhammad Altaf ${ }^{4}$, Rainer W. Bussmann ${ }^{5}$

${ }^{1}$ Clybay research private limited -560114 , Bangalore, India

2Department of Botany, University of Kashmir, Hazratbal, Srinagar - 190006, Jammu and Kashmir, India ${ }^{3}$ Centre of Research for Development/Environmental Sciences, Srinagar - 190006, Jammu and Kashmir, India ${ }^{4}$ Department of Forestry, Range and Wildlife management, The Islamia University of Bahawalpur, Pakistan

${ }^{5}$ Department of Ethnobotany, Institute of Botany, Ilia State University, 0105 Tbilisi, Georgia

*Corresponding Author: umerraj6668@gmail.com

Ethnobotany Research \& Applications 22:05 (2021)

\section{Databases and Inventories}

\begin{abstract}
Background: Cultural diversity in the Himalayan Mountain regions is closely linked to biodiversity, as there is a very close relationship between the local fauna and cultures. Religious rules and rituals also reinforce this relationship. Research has proven that these relationships are complex and differ based on a variety of factors.

Methods: There has been almost no research to explain the animal human interaction and commercially important animal species in different ethnic communities of Jammu and Kashmir. The present study was conducted throughout 2019-2020 and data were gathered through open and closed-end semi-structured interviews and group discussions.

Results: We found 10 species of mammals, 7 species of birds, and 7 species of fish were commercially important and playing a vital role in the economy of the local ethnic communities (Kashmiri, Pahari, Hanji, Bakarwal, Changapa). Among the documented species, seven species were unique to Hanji, followed by 4 species to Pahari, 3 to Kashmiri and 2 species to Changapa. The heat map classified predators into three groups that were recognized based on indicator species. Eight main predators (Selenarctos thibetanus, Vulpes vulpes, Herpestes auropunctatus, Canis familiaris, Felis catus, Canis lupus, Panthera uncia, and Panthera pardus) preying the documented species were recorded across the entire study area. Bos taurus primigenius, Bos Taurus and Gallus gallus domesticus played a significant role in the cultural and religious ritual aspects, whereas Capra aegagrus hircus, Equus ferus caballus and Gallus gallus domesticus were commonly used as a livelihood source among local communities.
\end{abstract}

Conclusions: Present study enlightened the interaction between the local fauna and cultures, meanwhile the study is first in its kind to document the livestock species with commercial value and specific communities associated with them in the former state Jammu and Kashmir.

Keywords: Commercial species; Economy; Ethnic groups, Jammu and Kashmir 


\section{Background}

Since the beginning of human civilization, animal resources have held great importance for human life with different animal species used in medicine (Saleem et al. 2021), diet (Rauf et al. 2017), faith (Alves et al. 2012), and a source of livelihood (Alves et al. 2012; Mughal et al. 2020). Working with animals is beneficial to one's well-being (Beetz et al. 2012), and they have an important role in the economy.

The National Research Council of the National Academies (2015) called for the food animal industry to prepare for larger food consumption demands that are expected to arise from a growing global population. Currently there are over 7.6 billion people in the world (United States Census Bureau2020) and many of them rely on the animal protein provided by livestock. The per head supply of red meat, poultry, and fish totaled 65,4kg in the United States, each with comparative percentages of 51\%, 42\%, and 7\%, respectively in 2017 (Bentley 2019). According to the United Nations (2019) the current world population will be increasing to 9.7 billion people by 2050. Meeting the nutritional needs of this population sustainably and furnishing its demand for animal products will require significant research and development $(R \& D)$ so that the present productivity can be sufficiently enhanced to meet the much-heightened future demands (National Research Council of the National Academies 2015).

India is an agrarian nation with a total of $11.6 \%$ of the world's livestock, the Indian livestock sector stays one of the largest in the world. (Shanmathy et al. 2018). The number of animals slaughtered for meat consumption includes cattle (3.05 million), buffalo (11.9 million), sheep (50.8 million), goat (97.2 million), and poultry (2.81 billion) (livestock-animal-production-statistics-of-India-2019/). India has surpassed China as the world's largest milk producer, accounting for $20.17 \%$ of global milk production. India produces about $5.65 \%$ of the world's eggs, $3 \%$ of the world's beef, and has the world's largest population of dairy animals (Shanmathy et al. 2018). The latest data from "National Dairy Development Board" indicate that there is a total of 535.8 million livestock and 851.8 million poultry in India (NDDB. Gov. of India 2020), and that the country currently has an annual fish production of about 9.06 million metric tons, holding second place in the world in total fish production (Fisheries and aquaculture Gov. of India 2020).

Jammu and Kashmir, including Ladakh, have a valuable livestock resource in the form of cattle, buffalo, sheep, goats, poultry, and other animals. The states estimated livestock population is 160.407 million, with 31.569 million cattle, 39.204 million sheep, 7.889 million buffaloes, 18.136 million goats, 58.311 million fowls, and 5.298 million ducks (Mir et al.2016). Grasslands, meadows, woodland, waste, barren, and fallow make up $44 \%$ (1061000 ha) of the total area of the state (2416000 ha) (Digest of Statistical2003-04 GO J\&K). Around 70\% of the population lives in rural areas and works in agriculture and related industries, such as livestock rearing. These people belong to different ethnic communities which are facing backwardness in economy and other aspects. The most common ethnic communities associated with livestock, are Gujjar, Pahari, Bakarwal, however Hanji community is only one associated with fish (Gairola et al. 2014). The number of livestock units per 1000 human population in the state is 736 animals, compared with a national average of 409 animals/1000 human population (Ahmad et al.2018). The estimated livestock population (excluding poultry) of the state is 9200842 which shares about 1.78 percent of the country's total livestock population. Traditionally specific tribes or communities are known for specific animals as their source of livelihoods. In the present study, our primary aims were to document the livestock species with commercial value and ethnic communities associated with them to form a base line study which can assist the stake holders to draft policies for overcoming the economic challenges faced by the said ethnic communities residing in the remote areas.

\section{Material and Methods Study Area}

Jammu and Kashmir (Fig.1), formerly one of the largest princely states of India and recently bifurcated into two union territories (Jammu \& Kashmir and Ladakh) by Central Government of India, is bordered to the northeast by the Uygur Autonomous Region of Xinjiang (China), to the east by the Tibet Autonomous Region (China), and the Chinese-administered portions of Kashmir, to the south by the Indian states of Himachal Pradesh and Punjab, to the southwest by Pakistan, and the northwest by the Pakistani-administered portion of Kashmir. It has a geographic area of $281382 \mathrm{~km}^{2}$. Inhabiting ethnic communities include Chowpan, Gujjar, Bakarwal, Dard, Kashmiri, Pathan, Pahari (Gairola et al. 2014). The communities mostly associated with commercial livestock species in Kashmir and Jammu regions are Kashmiri, Gujjar, Pahari, Hanji, and Bakarwal. However, Changapa is the only ethnic group associated with livestock in Ladakh. The various languages spoken by these ethnic groups are Urdu, Kashmiri, 
Pahari, Changskhat and Gujjari. About $68.3 \%$ population follows the religion of Islam fallowed by Hinduism (28.4\%), Sikhism (1.9\%), Buddhism (0.9\%) and Christianity (0.3\%) (Gov. of Jammu and Kashmir 2020). For present study different documenting areas (Fig. 1) were selected based on rich ethnic population communities interacting with species having commercial value.

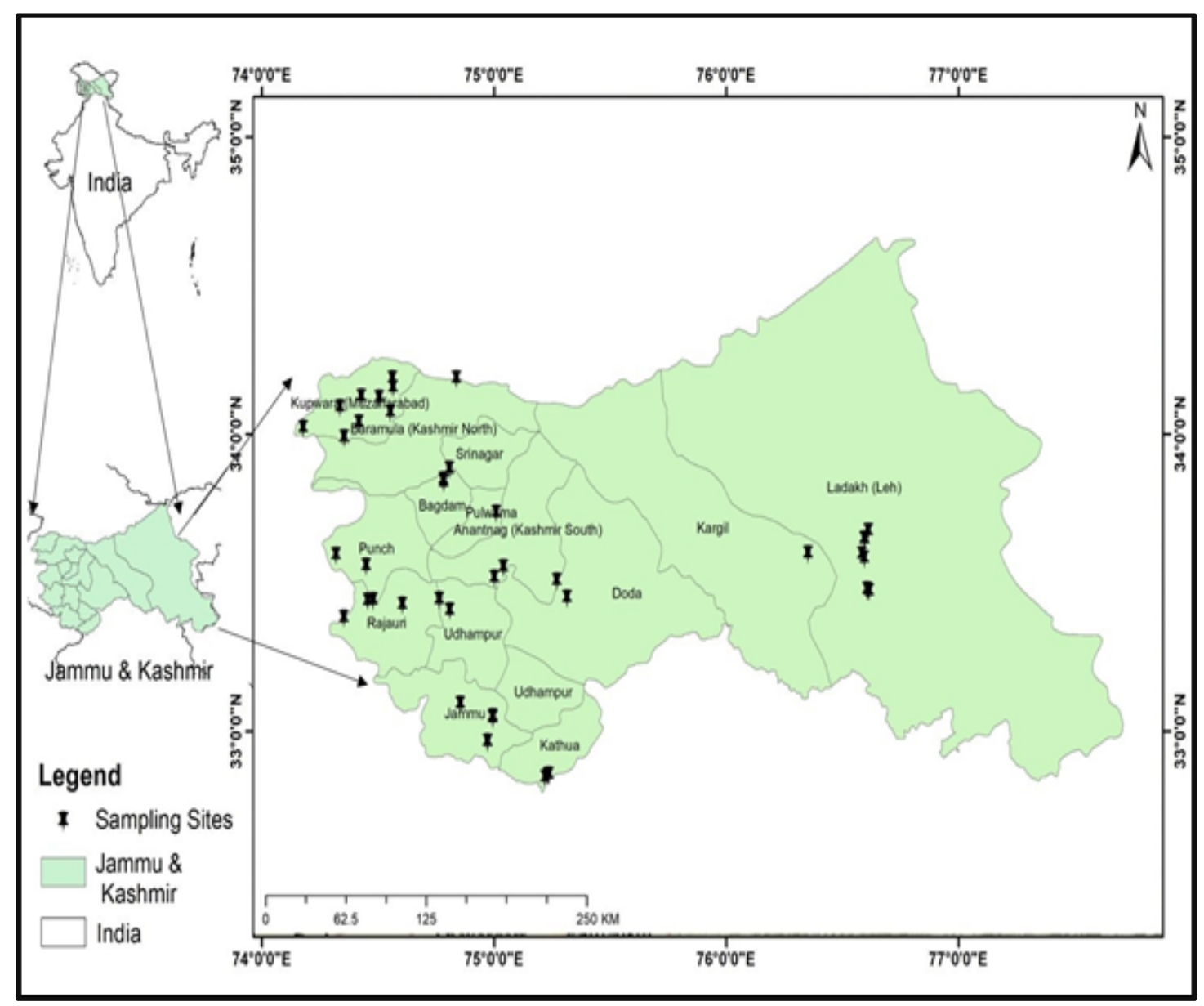

Figure 1. Map of Jammu and Kashmir, India and point showing survey sites in the erstwhile state Jammu and Kashmir.

\section{Survey and Data Collection}

The present study was conducted during 2019-2020. Data were collected through open and closed-end semistructured interviews and group discussions following Haq et al. (2020), after obtaining oral prior informed consent from the participants. The information was documented from the different groups of the study area, such as shepherds, herders, fishermen, cattlemen, and Local veterinary center staff (Table.1). Field-based personal observations, and additional information both informal and formal discussions along with photographs (Fig.2) were also conducted. The field study was carried out in diverse age-sex groups (young, old, and middle) (Table.1). The informants were also questioned for their views about the commercial species for self-consumption or livelihood generation. The collected data for (Table. 2) included the local name, use, cost, predators, feed, and fodder of the documented species.

\section{Data Analysis}

The questionnaire form was compiled in an ordered form and data was organized in Microsoft excel sheets and analyzed. Questionnaire data was initially analyzed for the basic categorization of the respondent's gender, age groups and literacy ratio, etc. Interaction analyses among animal species and predators was carried out by heatmap analysis. To produce the heat map, we used absence/presence data for showing the distribution of the species, and 
the analysis of clusters grouped the species having similar predators (Haq et al. 2020).The Sorensen's (Bray-Curtis) distance similarity coefficient based on presence/absence data was used for the identification of the significant differences among diverse animal species and predators (Sorensen 1948). The Venn diagram was created by using Bioinformatics \& Evolutionary Genomics software (Altaf et al. 2021). (http://bioinformatics.psb.ugent.be/cgibin/liste/Venn/calculate_venn.htpl).

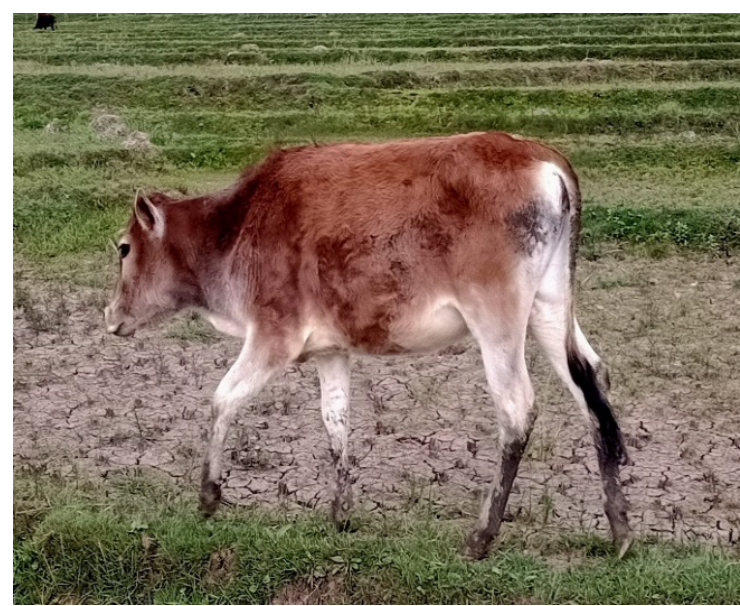

\section{Bos taurus}

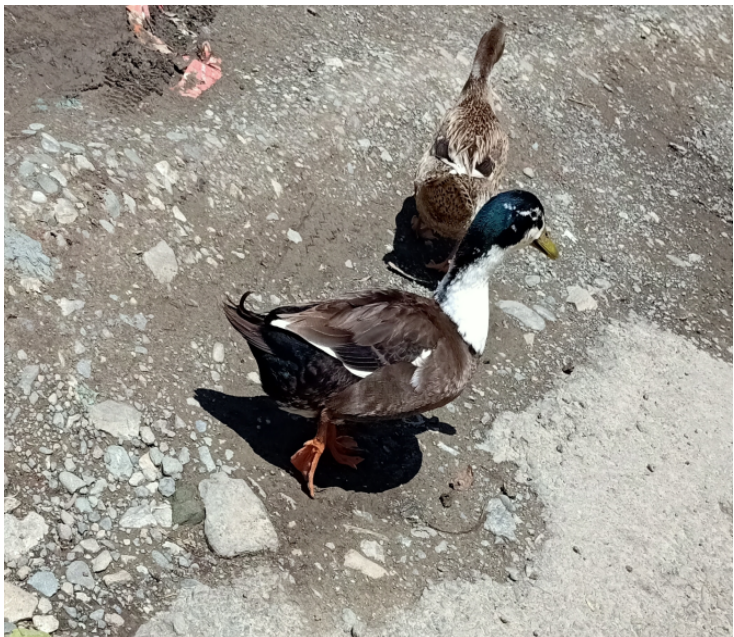

Anser platyrhynchos domesticus

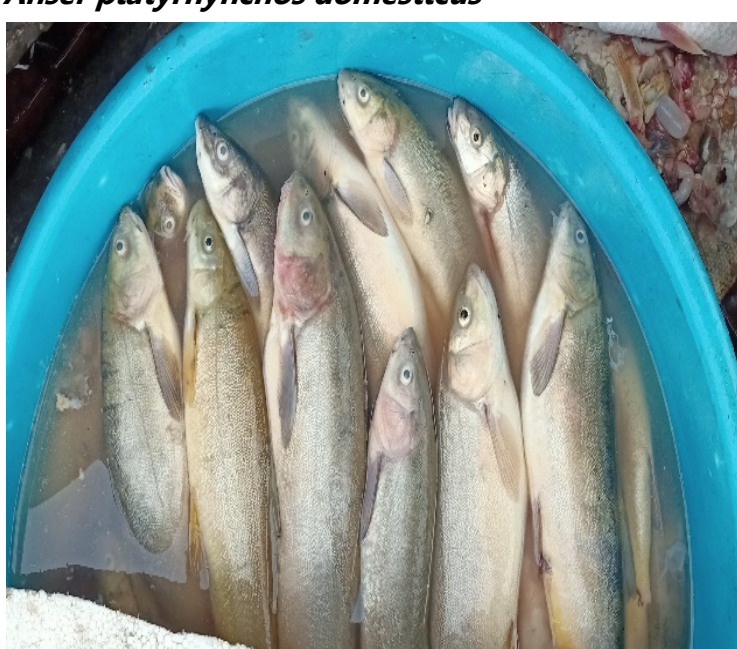

Schiothorax plagiostomus

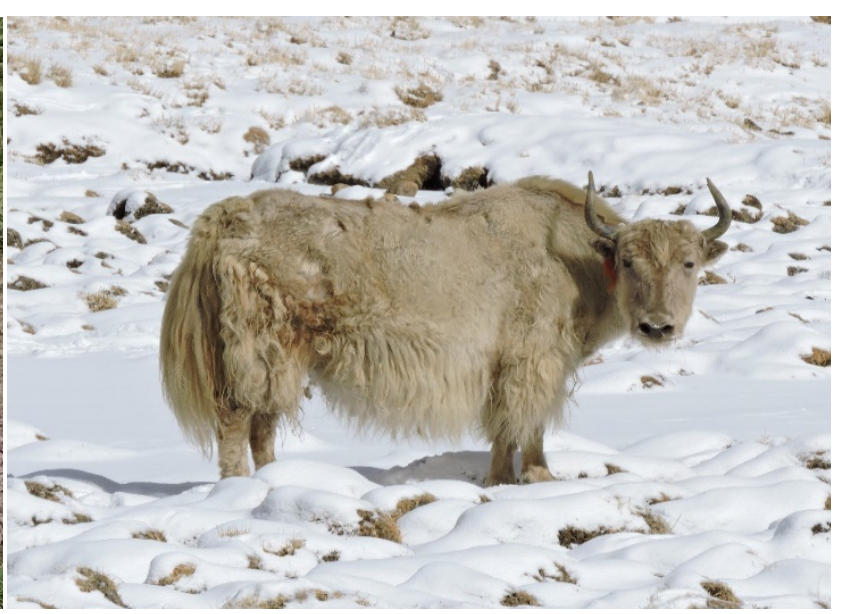

\section{Bos grunniens}

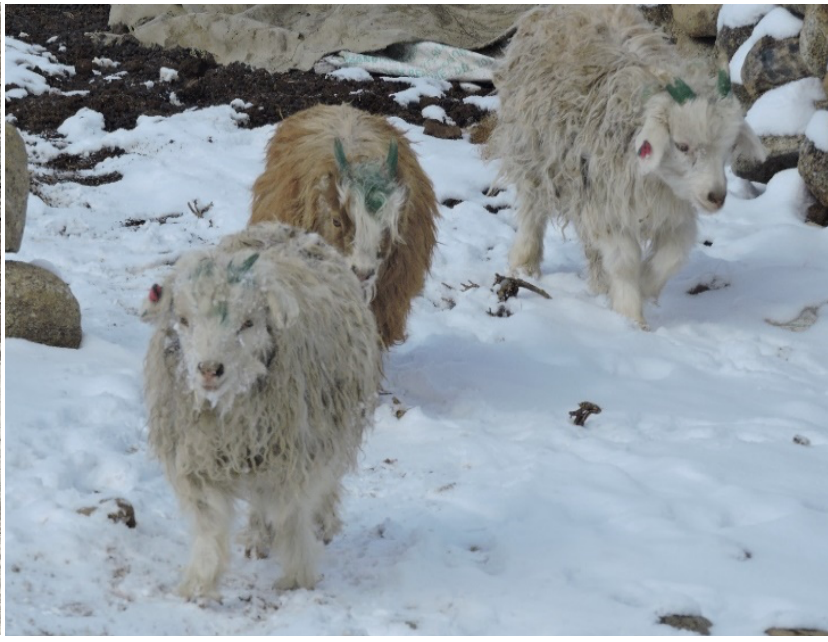

\section{Capra aegagrus hircus}

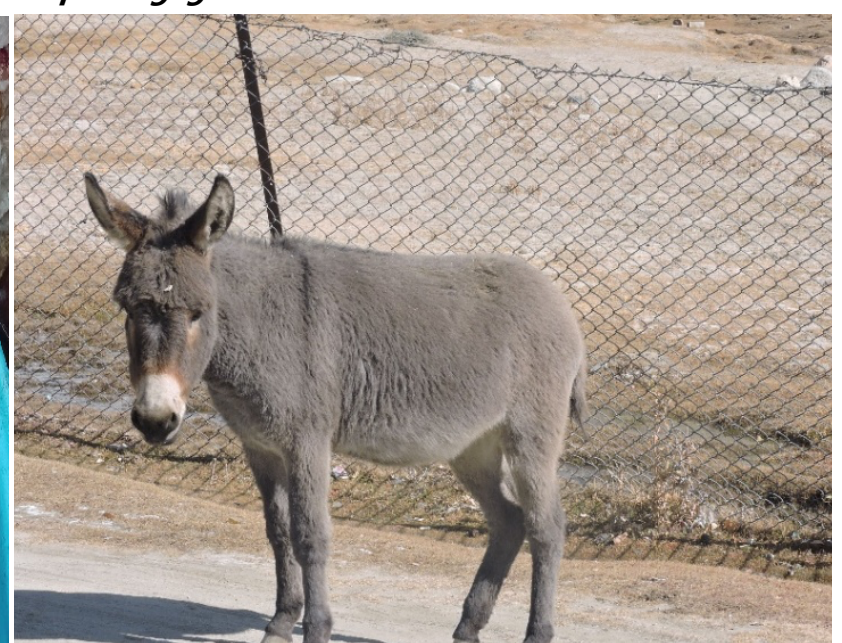

Equus africanus asinus

Figure 2. Photograph of few commercially important species sited during field study. 
Table 2. Documented commercial species across cultural group.

\begin{tabular}{|c|c|c|c|c|c|c|c|}
\hline Species & $\begin{array}{l}\text { Zoological name } \\
\text { Local name } \\
\text { Family }\end{array}$ & Habit & Usage & Predators & Natural Feed & $\begin{array}{l}\text { Market value } \\
\text { Owner value } \\
\text { Interconnector } \\
\text { value } \\
\text { (Indian rupees) } \\
\text { (Per kilogram) }\end{array}$ & $\begin{array}{l}\text { Ethnic } \\
\text { Groups }\end{array}$ \\
\hline \multicolumn{8}{|c|}{ Mammals } \\
\hline Ox & $\begin{array}{l}\text { Bos taurus primigenius } \\
\text { (Linnaeus, 1758) } \\
\text { Daand, } \\
\text { (Bovidae) }\end{array}$ & Herbivore & $\begin{array}{l}\text { Meat } \\
\text { offal } \\
\text { Dung } \\
\text { Ploughing }\end{array}$ & $\begin{array}{l}\text { Panthera pardus } \\
\text { (Leopard) } \\
\text { Selenarctos } \\
\text { thibetanus } \\
\text { (Black Bear) }\end{array}$ & $\begin{array}{l}\text { Natural pastures } \\
\text { Forest lands } \\
\text { Paddy grass } \\
\text { Tree leaves } \\
\text { Maize straw } \\
\text { Maize } \\
\text { Community lands }\end{array}$ & $\begin{array}{l}300 \\
220 \\
250\end{array}$ & $\begin{array}{l}\text { Kashmiri } \\
\text { Bakarwa } \\
\text { Pahari }\end{array}$ \\
\hline Cow & $\begin{array}{l}\text { Bos taurus } \\
\text { (Linnaeus, 1758) } \\
\text { Gaav, Gaan } \\
\text { (Bovidae) }\end{array}$ & Herbivore & $\begin{array}{l}\text { Meat } \\
\text { offal } \\
\text { Dung } \\
\text { Milk }\end{array}$ & $\begin{array}{l}\text { Panthera pardus } \\
\text { (Leopard) } \\
\text { Selenarctos } \\
\text { thibetanus } \\
\text { (Black Bear) }\end{array}$ & $\begin{array}{l}\text { Natural pastures } \\
\text { Forest lands } \\
\text { Paddy grass } \\
\text { Tree leaves } \\
\text { Maize straw } \\
\text { Maize } \\
\text { Community lands }\end{array}$ & $\begin{array}{l}300 \\
220 \\
250\end{array}$ & $\begin{array}{l}\text { Kashmiri } \\
\text { Pahari } \\
\text { Bakarwa }\end{array}$ \\
\hline Buffalo & $\begin{array}{l}\text { Bubalus bubalis } \\
\text { (Linnaeus, 1758) } \\
\text { Meains }^{\circ}, \text { Kata }^{\top}, \text { Meensa }^{\circ} \\
\text { (Bovidae) }\end{array}$ & Herbivore & $\begin{array}{l}\text { Meat } \\
\text { offal } \\
\text { Dung } \\
\text { Milk }\end{array}$ & $\begin{array}{l}\text { Panthera pardus } \\
\text { (Leopard) } \\
\text { Selenarctos } \\
\text { thibetanus } \\
\text { (Black Bear) }\end{array}$ & $\begin{array}{l}\text { Natural pastures } \\
\text { Paddy grass } \\
\text { Tree leaves } \\
\text { Maize straw } \\
\text { Community lands }\end{array}$ & $\begin{array}{l}300 \\
220 \\
250\end{array}$ & Pahari \\
\hline Sheep & 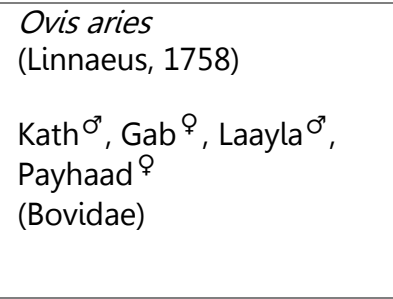 & Herbivore & $\begin{array}{l}\text { Meat } \\
\text { Pellets } \\
\text { Milk } \\
\text { Wool }\end{array}$ & $\begin{array}{l}\text { Panthera pardus } \\
\text { (Leopard) } \\
\text { Selenarctos } \\
\text { thibetanus } \\
\text { (Black Bear) } \\
\text { Herpestes } \\
\text { auropunctatus } \\
\text { (Mangoos) }\end{array}$ & $\begin{array}{l}\text { Tree leave } \\
\text { Natural pastures } \\
\text { Community lands }\end{array}$ & $\begin{array}{l}500 \\
300 \\
380\end{array}$ & $\begin{array}{l}\text { Kashmiri } \\
\text { Pahari } \\
\text { Bakarwa } \\
\text { Changpa }\end{array}$ \\
\hline
\end{tabular}


Ethnobotany Research and Applications

\begin{tabular}{|c|c|c|c|c|c|c|c|}
\hline Goat & $\begin{array}{l}\text { Capra hircus } \\
\text { (Linnaeus, 1758) } \\
\text { Chaavaj }^{\circ}, \text { Chaavul }^{\circ}, \\
\text { Bakrie }^{9}, \text { Bkra }^{0^{\prime \prime}} \\
\text { (Bovidae) }\end{array}$ & Herbivore & $\begin{array}{l}\text { Meat } \\
\text { Pellets } \\
\text { Milk } \\
\text { Wool }\end{array}$ & $\begin{array}{l}\text { Panthera pardus } \\
\text { (Leopard) } \\
\text { Selenarctos } \\
\text { thibetanus } \\
\text { (Black Bear) } \\
\text { Herpestes } \\
\text { auropunctatus } \\
\text { (Mangoos) }\end{array}$ & $\begin{array}{l}\text { Tree leave } \\
\text { Natural pastures } \\
\text { Community lands }\end{array}$ & $\begin{array}{l}300 \\
380 \\
500\end{array}$ & $\begin{array}{l}\text { Kashmiri } \\
\text { Pahari } \\
\text { Bakarwal }\end{array}$ \\
\hline Horse ${ }^{* * *}$ & 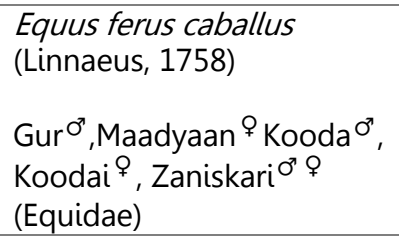 & Herbivore & $\begin{array}{l}\text { Chariot } \\
\text { (Tanga, Rada) }\end{array}$ & $\begin{array}{l}\text { Panthera pardus } \\
\text { (Leopard) } \\
\text { Selenarctos } \\
\text { thibetanus } \\
\text { (Black Bear) }\end{array}$ & $\begin{array}{l}\text { Tree leave } \\
\text { Natural pastures } \\
\text { Community lands } \\
\text { Paddy grass } \\
\text { Tree leaves }\end{array}$ & $\begin{array}{l}60000 \\
57000 \\
58000\end{array}$ & $\begin{array}{l}\text { Kashmiri } \\
\text { Pahari } \\
\text { Bakarwal } \\
\text { Changpa }\end{array}$ \\
\hline Donkey*** & 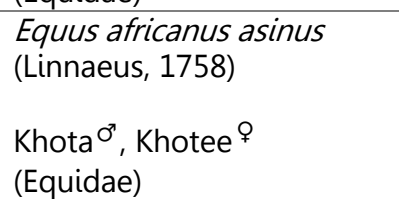 & Herbivore & $\begin{array}{l}\text { Beast of } \\
\text { burden }\end{array}$ & $\begin{array}{l}\text { Panthera pardus } \\
\text { (Leopard) } \\
\text { Selenarctos } \\
\text { thibetanus } \\
\text { (Black Bear) }\end{array}$ & $\begin{array}{l}\text { Tree leave } \\
\text { Natural pastures } \\
\text { Community lands } \\
\text { Paddy grass }\end{array}$ & $\begin{array}{l}25000 \\
23000 \\
24000\end{array}$ & $\begin{array}{l}\text { Pahari } \\
\text { Bakarwal }\end{array}$ \\
\hline Camel & $\begin{array}{l}\text { Camelus dromedarius } \\
\text { (Linnaeus, 1758) } \\
\text { Oohont }^{0}, \text { Oonntdni }^{\circ} \\
\text { (Camelidae) }\end{array}$ & Herbivore & Meat & $\begin{array}{l}\text { Panthera pardus } \\
\text { (Leopard) } \\
\text { Selenarctos } \\
\text { thibetanus } \\
\text { (Black Bear) }\end{array}$ & $\begin{array}{l}\text { Tree leave } \\
\text { Natural pastures } \\
\text { Community lands } \\
\text { Paddy grass }\end{array}$ & $\begin{array}{l}300 \\
180 \\
230\end{array}$ & Pahari \\
\hline Pashmina goat & 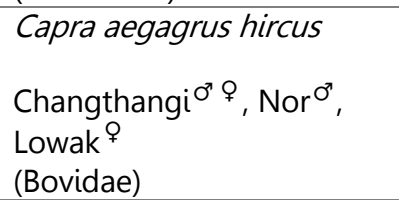 & Herbivore & $\begin{array}{l}\text { Meat } \\
\text { Pashmina } \\
\text { Milk }\end{array}$ & $\begin{array}{l}\text { Panthera pardus } \\
\text { (Leopard) } \\
\text { Selenarctos } \\
\text { thibetanus } \\
\text { (Black Bear) }\end{array}$ & $\begin{array}{l}\text { Tree leave } \\
\text { Natural pastures } \\
\text { Community lands } \\
\text { Paddy grass } \\
\text { Forest lands }\end{array}$ & $\begin{array}{l}600 \\
320 \\
420\end{array}$ & Changpa \\
\hline Yak & $\begin{array}{l}\text { Bos grunniens } \\
\text { (Linnaeus, 1766) } \\
\text { Yak, Dri, Nak } \\
\text { (Bovidae) }\end{array}$ & Herbivore & $\begin{array}{l}\text { Milk } \\
\text { Meat } \\
\text { Wool }\end{array}$ & $\begin{array}{l}\text { Panthera pardus } \\
\text { (Leopard) } \\
\text { Selenarctos } \\
\text { thibetanus } \\
\text { (Black Bear) }\end{array}$ & $\begin{array}{l}\text { Tree leave } \\
\text { Natural pastures } \\
\text { Community lands } \\
\text { Forest lands }\end{array}$ & $\begin{array}{l}350 \\
200 \\
250\end{array}$ & Changpa \\
\hline
\end{tabular}


Ethnobotany Research and Applications

\begin{tabular}{|c|c|c|c|c|c|c|c|}
\hline \multicolumn{8}{|l|}{ Birds } \\
\hline Duck & $\begin{array}{l}\text { Anas platyrhynchos } \\
\text { domesticus } \\
\text { Bataich }^{\circ}, \text { Baatuk }^{\circ} \\
\text { (Anatidae) }\end{array}$ & Omnivore & $\begin{array}{l}\text { Egg } \\
\text { Meat } \\
\text { Droppings }\end{array}$ & $\begin{array}{l}\text { Herpestes } \\
\text { auropunctatus } \\
\text { (Mangoos) } \\
\text { Vulpes vulpes } \\
\text { (Fox) }\end{array}$ & $\begin{array}{l}\text { Maize } \\
\text { Paddy } \\
\text { Small Insects } \\
\text { Aquatic weeds }\end{array}$ & $\begin{array}{l}620 \\
350 \\
500\end{array}$ & Kashmiri \\
\hline Goose & $\begin{array}{l}\text { Anser anser domesticus } \\
\text { Anz }{ }^{\circ} \\
\text { (Anatidae) }\end{array}$ & Omnivore & $\begin{array}{l}\text { Egg } \\
\text { Meat } \\
\text { Droppings }\end{array}$ & $\begin{array}{l}\text { Herpestes } \\
\text { auropunctatus } \\
\text { (Mangoos) } \\
\text { Vulpes vulpes } \\
\text { (Fox) }\end{array}$ & $\begin{array}{l}\text { Maize } \\
\text { Paddy } \\
\text { Small Insects } \\
\text { Aquatic weeds }\end{array}$ & $\begin{array}{l}700 \\
500 \\
550\end{array}$ & Kashmiri \\
\hline Rooster & $\begin{array}{l}\text { Gallus gallus domesticus } \\
\text { (Linnaeus, 1758) } \\
\text { Kukur, Kukud } \\
\text { (Phasianidae) }\end{array}$ & Omnivore & $\begin{array}{l}\text { Meat } \\
\text { Droppings }\end{array}$ & $\begin{array}{l}\text { Vulpes vulpes } \\
\text { (Fox) }\end{array}$ & $\begin{array}{l}\text { Maize } \\
\text { Paddy } \\
\text { Small Insects }\end{array}$ & $\begin{array}{l}550 \\
400 \\
460\end{array}$ & $\begin{array}{l}\text { Kashmiri } \\
\text { Pahari }\end{array}$ \\
\hline Hen & $\begin{array}{l}\text { Gallus gallus domesticus } \\
\text { (Linnaeus, 1758) } \\
\text { Kakar, Kukadi } \\
\text { (Phasianidae) }\end{array}$ & Omnivore & $\begin{array}{l}\text { Egg } \\
\text { Meat } \\
\text { Droppings }\end{array}$ & $\begin{array}{l}\text { Vulpes vulpes } \\
\text { (Fox) }\end{array}$ & $\begin{array}{l}\text { Maize } \\
\text { Paddy } \\
\text { Small Insects }\end{array}$ & $\begin{array}{l}450 \\
350 \\
370\end{array}$ & $\begin{array}{l}\text { Kashmiri } \\
\text { Pahari }\end{array}$ \\
\hline $\begin{array}{l}\text { Kashmiri jungle } \\
\text { fowl }\end{array}$ & $\begin{array}{l}\text { Gallus sonneratii (Temminck, } \\
\text { 1813) } \\
\text { Bndkukud }{ }^{\text {ơ }} 9 \\
\text { (Phasianidae) }\end{array}$ & Omnivore & Meat & $\begin{array}{l}\text { Herpestes } \\
\text { auropunctatus } \\
\text { (Mangoos) } \\
\text { Vulpes vulpes } \\
\text { (Fox) }\end{array}$ & $\begin{array}{l}\text { Maize } \\
\text { Paddy } \\
\text { Small Insects }\end{array}$ & $\begin{array}{l}1000 \\
600 \\
800\end{array}$ & Pahari \\
\hline Broiler & $\begin{array}{l}\text { Gallus gallus domesticus } \\
\text { Boiler } \\
\text { (Phasianidae) }\end{array}$ & Omnivore & $\begin{array}{l}\text { Meat } \\
\text { Droppings }\end{array}$ & $\begin{array}{l}\text { Herpestes } \\
\text { auropunctatus } \\
\text { (Mangoos) } \\
\text { Vulpes vulpes } \\
\text { (Fox) }\end{array}$ & $\begin{array}{l}\text { Maize } \\
\text { Paddy } \\
\text { Small Insects }\end{array}$ & $\begin{array}{l}160 \\
100 \\
130\end{array}$ & $\begin{array}{l}\text { Kashmiri } \\
\text { Pahari }\end{array}$ \\
\hline Hill Pigeon** & $\begin{array}{l}\text { Columba rupestris } \\
\text { (Pallas, 1811) } \\
\text { Koturơ } \$ \\
\text { (Columbidae) }\end{array}$ & Omnivore & Meat & $\begin{array}{l}\text { Vulpes vulpes } \\
\text { (Fox) }\end{array}$ & $\begin{array}{l}\text { Maize } \\
\text { Paddy } \\
\text { Small Insects } \\
\text { Natural pastures } \\
\text { Forest lands }\end{array}$ & $\begin{array}{l}20000 \\
19000 \\
18000\end{array}$ & Kashmiri \\
\hline
\end{tabular}


Ethnobotany Research and Applications

\begin{tabular}{|c|c|c|c|c|c|c|c|}
\hline \multirow{2}{*}{$\begin{array}{l}\text { Fish } \\
\text { Alghad Snow } \\
\text { trout }\end{array}$} & & & & & & & \\
\hline & $\begin{array}{l}\text { Schizopyge niger } \\
\text { (Heckel, 1838) } \\
\text { Ale Gad } \\
\text { (Cyprinidae) }\end{array}$ & Herbivore & $\begin{array}{l}\text { Meat } \\
\text { Fish waste }\end{array}$ & $\begin{array}{l}\text { Selenarctos } \\
\text { thibetanus } \\
\text { (Black Bear) }\end{array}$ & $\begin{array}{l}\text { Phytoplankton's } \\
\text { Aquatic weeds }\end{array}$ & $\begin{array}{l}450 \\
200 \\
300\end{array}$ & Hanji \\
\hline Hill trout & $\begin{array}{l}\text { Schizothorax plagiostomus } \\
\text { (Heckel, 1838) } \\
\text { Khont } \\
\text { (Cyprinidae) }\end{array}$ & Herbivore & $\begin{array}{l}\text { Meat } \\
\text { Fish waste }\end{array}$ & $\begin{array}{l}\text { Selenarctos } \\
\text { thibetanus } \\
\text { (Black Bear) }\end{array}$ & $\begin{array}{l}\text { Phytoplankton's } \\
\text { Aquatic weeds }\end{array}$ & $\begin{array}{l}400 \\
180 \\
250\end{array}$ & Hanji \\
\hline $\begin{array}{l}\text { Chirruh Snow } \\
\text { trout }\end{array}$ & $\begin{array}{l}\text { Schizothorax esocinus } \\
\text { (Heckel, 1838) } \\
\text { Chhurru } \\
\text { (Cyprinidae) }\end{array}$ & Herbivore & $\begin{array}{l}\text { Meat } \\
\text { Fish waste }\end{array}$ & $\begin{array}{l}\text { Selenarctos } \\
\text { thibetanus } \\
\text { (Black Bear) }\end{array}$ & $\begin{array}{l}\text { Phytoplankton's } \\
\text { Aquatic weeds }\end{array}$ & $\begin{array}{l}400 \\
200 \\
300\end{array}$ & Hanji \\
\hline $\begin{array}{l}\text { Kunar Snow } \\
\text { trout }\end{array}$ & $\begin{array}{l}\text { Schizothorax labiatus } \\
\text { (McClelland, 1842) } \\
\text { Chush } \\
\text { (Cyprinidae) }\end{array}$ & Herbivore & $\begin{array}{l}\text { Meat } \\
\text { Fish waste }\end{array}$ & $\begin{array}{l}\text { Selenarctos } \\
\text { thibetanus } \\
\text { (Black Bear) }\end{array}$ & $\begin{array}{l}\text { Phytoplankton's } \\
\text { Aquatic weeds }\end{array}$ & $\begin{array}{l}400 \\
200 \\
300\end{array}$ & Hanji \\
\hline Common carp & $\begin{array}{l}\text { Cyprinus carpio } \\
\text { (Linnaeus, 1758) } \\
\text { Punjab Gad } \\
\text { (Cyprinidae) }\end{array}$ & Omnivore & $\begin{array}{l}\text { Meat } \\
\text { Fish waste }\end{array}$ & $\begin{array}{l}\text { Selenarctos } \\
\text { thibetanus } \\
\text { (Black Bear) }\end{array}$ & $\begin{array}{l}\text { Phytoplankton's } \\
\text { Aquatic weeds } \\
\text { Zooplanktons } \\
\text { Small Insects }\end{array}$ & $\begin{array}{l}350 \\
170 \\
250\end{array}$ & Hanji \\
\hline Brown trout & $\begin{array}{l}\text { Salmo truttas } \\
\text { (Linnaeus, 1758) } \\
\text { Punjab Gad } \\
\text { (Salmonidae) }\end{array}$ & Carnivore & $\begin{array}{l}\text { Meat } \\
\text { Fish waste }\end{array}$ & $\begin{array}{l}\text { Selenarctos } \\
\text { thibetanus } \\
\text { (Black Bear) }\end{array}$ & $\begin{array}{l}\text { Zooplanktons, } \\
\text { Small Insects }\end{array}$ & $\begin{array}{l}350 \\
200 \\
275\end{array}$ & Hanji \\
\hline Rainbow trout & $\begin{array}{l}\text { Oncorhynchus mykiss } \\
\text { (Walbaum, 1792) } \\
\text { Punjab Gad } \\
\text { (Salmonidae) }\end{array}$ & Omnivore & $\begin{array}{l}\text { Meat } \\
\text { Fish waste }\end{array}$ & $\begin{array}{l}\text { Selenarctos } \\
\text { thibetanus } \\
\text { (Black Bear) }\end{array}$ & $\begin{array}{l}\text { Phytoplankton's } \\
\text { Aquatic weeds } \\
\text { Zooplanktons } \\
\text { Small Insects }\end{array}$ & $\begin{array}{l}350 \\
200 \\
280\end{array}$ & Hanji \\
\hline
\end{tabular}

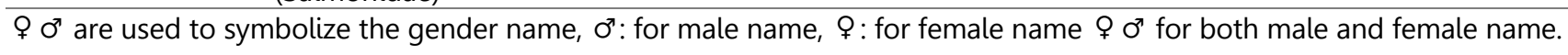

**Species are not valued as whole individuals. 


\section{Demographic details of respondents and their views about the commercial species}

We conducted interviews with 260 informants, of whom 93 were from Jammu, 119 from Kashmir, and 48 were from Ladakh. During the survey respondents comprised an uneven distribution of male-female ratio, where 205 were men, and 55 were women. The fewer number of female participants could be due to the reason that women remain indoor having less exposure to remote sites and many of them also showed reluctance (Haq et al. 2020a; Asif et al. 2021). Most of the respondents were old-aged (44.23\%), followed by middle aged (37.30\%), and young (18.46\%). More than half of the respondents were without formal education (63.84\%). The respondents interviewed included Shepherd's (33.70\%), Herders (21.53\%), Local veterinary centers (4.23\%), Fisherman (20\%), and Cattlemen (21.15\%). Selected respondents belonged to the four ethnic groups (Kashmiri, Hanji, Pahari, Bakarwal, Changapa) (Table 1).

Table 1. Demographic status of the respondents from the study area.

\begin{tabular}{|c|c|c|c|c|c|}
\hline \multirow[t]{2}{*}{ Demographic features } & \multirow{2}{*}{$\begin{array}{l}\text { Number of } \\
\text { people }\end{array}$} & \multirow[t]{2}{*}{ Percentage } & \multicolumn{3}{|c|}{ Bio-geographic provinces } \\
\hline & & & Jammu & Kashmir & Ladakh \\
\hline Ethnic groups & & & $\begin{array}{l}\text { Pahari } \\
\text { Bakarwal }\end{array}$ & $\begin{array}{l}\text { Kashmiri } \\
\text { Pahari } \\
\text { Bakarwal } \\
\text { Hanji }\end{array}$ & Changapa \\
\hline Religion & & & $\begin{array}{l}\text { Hinduism } \\
\text { Islam Sikhism }\end{array}$ & Islam Sikhism & $\begin{array}{l}\text { Tibetan } \\
\text { Buddhism }\end{array}$ \\
\hline Language & & & $\begin{array}{l}\text { Hindi } \\
\text { Pahari }\end{array}$ & $\begin{array}{l}\text { Kashmiri } \\
\text { Pahari }\end{array}$ & Changskhat \\
\hline Respondents & 260 & & $\begin{array}{l}93 \\
(35.76 \%)\end{array}$ & $\begin{array}{l}119 \\
(45.76 \%)\end{array}$ & $\begin{array}{l}48 \\
(18.46 \%)\end{array}$ \\
\hline \multicolumn{6}{|l|}{ Education } \\
\hline Illiterate & 166 & 63.84 & 59 & 73 & 34 \\
\hline Primary education & 50 & 19.23 & 16 & 25 & 9 \\
\hline Secondary education & 32 & 12.30 & 13 & 15 & 4 \\
\hline Higher education & 12 & 4.61 & 5 & 6 & 1 \\
\hline \multicolumn{6}{|l|}{ Age range } \\
\hline Young (18-26 Years) & 48 & 18.46 & 15 & 25 & 8 \\
\hline Middle (56-75 Years) & 97 & 37.30 & 33 & 45 & 19 \\
\hline Old (27-55 Years) & 115 & 44.23 & 45 & 49 & 21 \\
\hline \multicolumn{6}{|l|}{ Profession } \\
\hline Shepherds & 86 & 33.07 & 25 & 40 & 21 \\
\hline Herders & 56 & 21.53 & 18 & 23 & 15 \\
\hline Local veterinary centers & 11 & 4.23 & 3 & 7 & 1 \\
\hline Fisherman & 52 & 20.0 & 26 & 26 & 0 \\
\hline Cattlemen & 55 & 21.15 & 21 & 23 & 11 \\
\hline \multicolumn{6}{|l|}{ Gender } \\
\hline Male & 205 & 78.84 & 75 & 94 & 36 \\
\hline Female & 55 & 21.15 & 18 & 25 & 12 \\
\hline
\end{tabular}

A large portion (78\%) treated commercial species as the backbone of the rural economy. In contrast (13\%) believed that it's an orthodox source of income, society needs to come over and look into other means of income like real estate.

\section{Results}

\section{Classification of Documented species}

The present study documented 24 commercial species. Documented species were broadly classified into mammals (44\%), birds (28\%), and fish (28\%), (Fig. 3a) further classified based on nutrition most species were herbivores (60\%) followed by omnivores (36\%) and carnivores (4\%) (Fig. 3b). Altaf et al. (2017) reported the use of different animal 
species with commercial usage in Pakistan; meanwhile, the use of fish in different cultures was also reported in the other parts of the Himalayas (Altaf et al. 2018).

\section{Unique and Common Species Across Cultures}

The Venn diagram (Fig. 4) shows that 7 species (Schizothorax esocinus, Cyprinus carpio, Schizothorax labiatus, Schizo pygeniger, Oncorhynchus mykiss, Salmo trutta Schizothorax plagiostomus), were unique to the Hanji ethnic group, followed by 3 species (Bubalus bubalis, Camelus dromedarius, Gallus sonneratii) unique to Pahari, 3 species (Anas platyrhynchos, Columba rupestris, Anser anser domesticus) unique to Kashmiri, 2 species (Bos grunniens, Capra aegagrus hircus) unique to Changapa. However, 3 species (Gallus gallus domesticus (rooster), Gallus gallus domesticus (broiler), Gallus gallus domesticus (hen) were found common in Kashmiri and Pahari followed by 2 species (Bos taurus primigenius, Equus africanus asinus) common in Bakarwal and Pahari, 2 species (Bos taurus, Capra hircus) in Bakarwal, Kashmiri and Pahari, 2 species (Ovis aries, Equus ferus caballus) in Bakarwal, Changapa, Kashmiri and Pahari. Similar use of species (animals, birds, fish) across different ethnic groups were also reported by Altaf et al. (2017); Altaf et al. (2018) from Pakistan Himalaya.
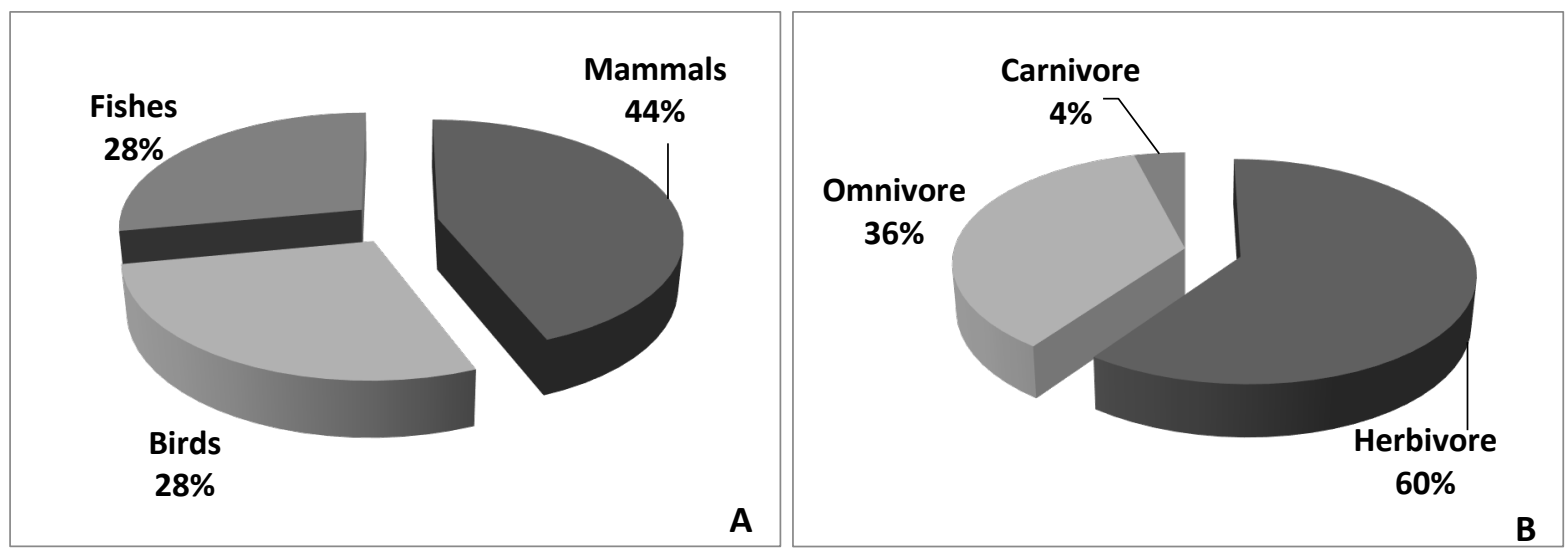

Figure 3. (A) Percentage of documented species. (B) Percentage of documented species based on nutrition.

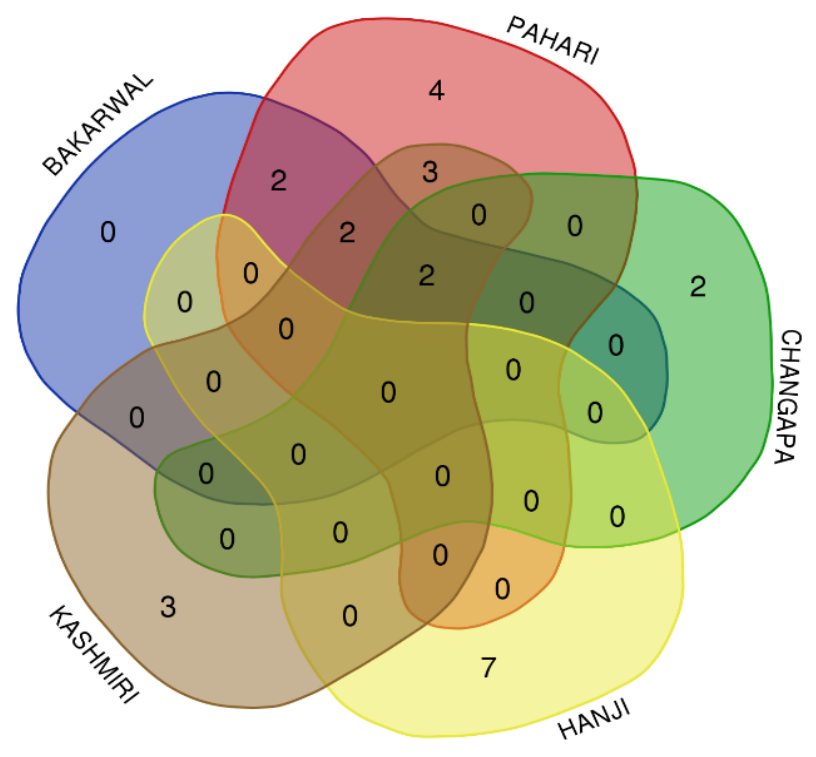

Figure 4. Venn diagram representing species overlap across different ethnic communities.

\section{Diversity and Usage Across Ethnic Communities Species and usage with respect to Kashmiri}

A total of 11 commercial species were recorded, of which 5 species (ox, cow, sheep, goat, horse) belonged to mammals, 6 belonged to birds (duck, rooster, goose, broiler, hen, hill pigeon). These species belonged to a variety of families and the dominant families were Phasianidae $(\mathrm{N}=3)$, Bovidae $(\mathrm{N}=3)$ (Table 2). Similarly, Mahawar and Jaroli (2007) reported various animals with the commercial value from Rajasthan - India. Meat was the prime part used as food followed by offal. Globally meat is considered a rich source of protein and poultry meat production has increased by $1,144 \%$ in the last 50 years (Hoffman and Falvo 2004).

Horse is used for transport i.e.: Horses are tied to a chariot-like carriage locally called Tanga (Fig. 5) which carries passengers. Meanwhile, species like Sheep, and Goat were used mostly for milk and wool apart from meat. Wool obtained from the Sheep is used for making shawls and blankets. Earlier a hand-made blanket was made in the valley (Kashmir) from wool called Chaadar. Now Chaadar making has vanished due to the availability of low-cost 
blankets in the market, along with this their pellets from Sheep and Goat are used in orchid cultivation as manure, similarly, the dung from Cow, Ox and the droppings of rooster, hen, and broiler are also used as organic fertilizers. Dung is first decomposed in a pit for more than a year and then used. Duck and Goose are used to lay eggs. Hill pigeon is mainly used as a recreational bird and sometimes also used for bush meat. Ox is used for ploughing the field (Table 2). Likewise, Altaf et al. (2017) also reported the interaction levels between animal species and humans from Pakistan which is having the geographical contiguity with Jammu and Kashmir.

\section{Species and usage with respect to Pahari}

The Pahari community mentioned 13 commercial species, among which 8 species, (ox, cow, buffalo, sheep, goat, horse, donkey, and camel) belonged to mammals and 4 species (rooster, hen, Kashmiri jungle fowl, broiler) belonged to birds, no fish species were recorded. Likewise, the Kashmiri, meat was the mostly consumed, followed by offal. Most species belonged to Bovidae $(\mathrm{N}=5)$ followed by Phasianidae $(\mathrm{N}=4)$ (Table 2). Species like cow, sheep and goat are primarily used for milk. Sheep and goat are also used for wool. Camel is used mainly for meat, hen for eggs. Horse and donkey are used as beasts of burden and except for these two species all others are used for meat. Kashmiri jungle fowl is consumed as food.

The Pahari community is highly marginalized community. Many of them are seen in upper hilly areas and have a strong relation with their animal fauna as they use them in agricultures, also trade them and earn the livelihood. Jina et al. (1996) reported usage of animal fauna in the former Jammu and Kashmir.

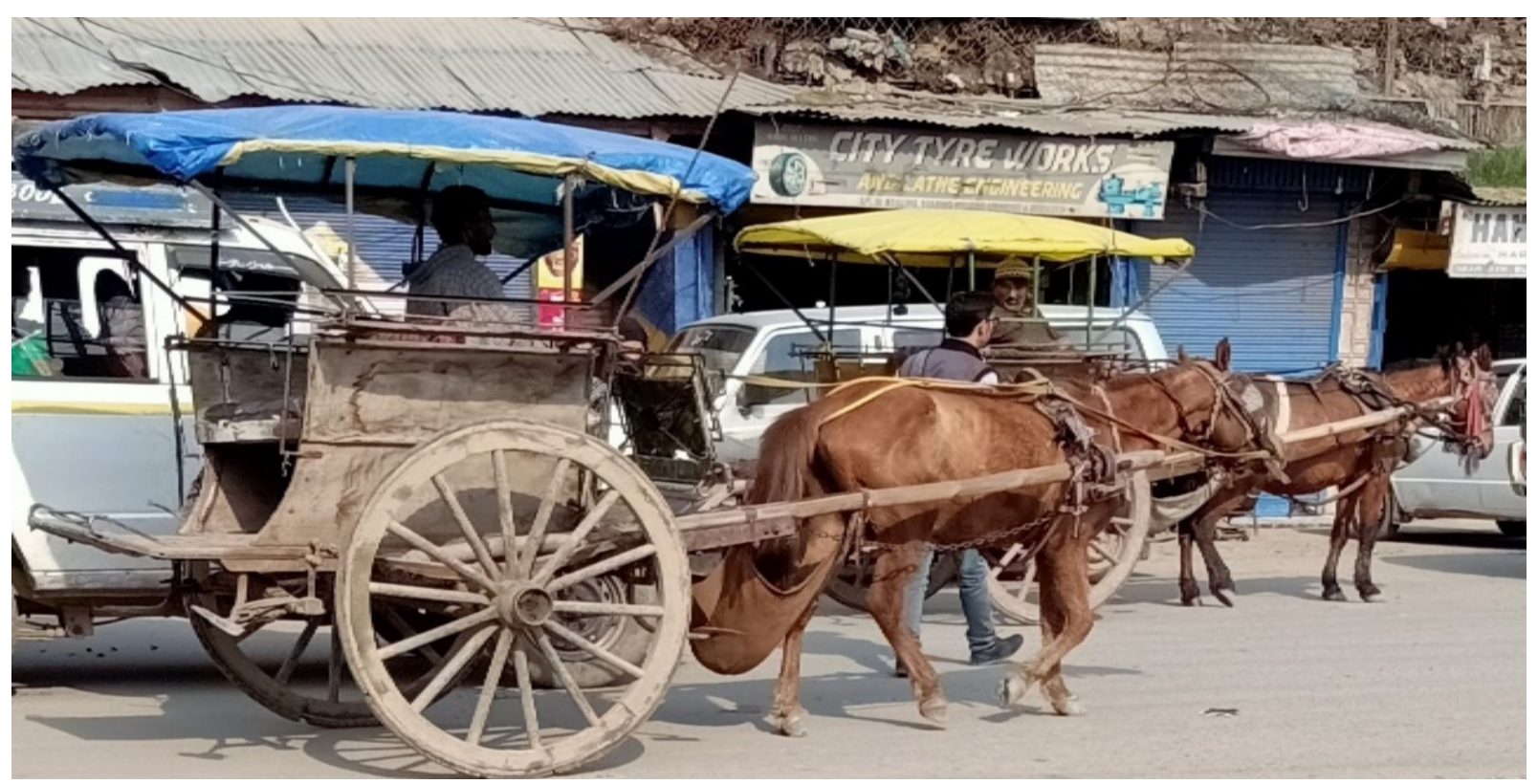

Figure 5. Chariot-like carriage called Tanga

\section{Species and usage with respect to Bakarwal}

The nomadic Bakarwal are mostly dependent on livestock. We documented six species (cow, ox, sheep, goat, horse, and donkey) from this ethnic group. All recorded species belonged to mammals, with 4 species (cow, ox, sheep, and goat) from Bovidae and 2 species (horse and donkey) from Equidae (Table 2). The documented species are a real asset to the ethnic community as they are permanently dependent on these species for survival and lacking modern amenities creating a bond at social, economic, and emotional levels. Species like sheep, goat and cow are used for milk and often meat, at the same time these species are sold for money. Dutta et al. (2021) also reported the dependence of Bakarwal on livestock and lack of modern conveniences. In Jammu and Kashmir, this tribe migrates seasonally from Jammu province to Kashmir and vice versa to avoid unavailability of feed and to protect from the harsh climate. 


\section{Species and usage with respect to Changapa}

The Changapa from Ladakh has few resources for survival and is mainly nomadic like the Bakarwal. The present study documented 4 commercial species (sheep, horse, yak and Changthangi (pashmina goat)) from mammals, among which 3 species (sheep, Changthangi, and yak) belonged to the family Bovidae and 1 species (horse) belonged to Equidae, the ethnic group mainly used these species for milk, wool, (pashmina) (Table2), and in few cases for meat. This tribe is dependent on the livestock, and milk is praised and considered an elixir. Milk is composed of organic compounds including fat, protein, carbohydrates, water, and solids particles (Hamad and Baiomy 2010; Merlin et al. 2015) and meets almost all nutritional requirements. Sheep is used mainly for meat during winters and the Changthangi is used for wool, (Pashmina, Pashm).

Meat composition is a different cause of the impacts of different factors like feed, water, breed, sex, etc. (Keeton and Eddy 2004; Hui 2012; Cheung and Mehta 2015; Haidar and Bashir 2021). Jina et al. (1996) reported the use of sheep for meat and Changthangi for Pashmina (Pashm). It is believed that Pashmina is the finest fiber of all goat hair. The milk of Changthangi is also highly praised and is believed to give strength to the body and protect from jaundice and cough. Chellappandian et al. (2014) also reported its use against jaundice from Tamil Nadu in India. Mola et al. (2020) reported the use of milk against cough in Ethiopia.

The Changapa live in challenging conditions (harsh climate) without modern facilities and the above said species are only to assist for overcoming these harsh, challenging environmental conditions creating a unique animal human bond in the said region of study area. Martos Martinez-Caja (2021) also discussed the humans and animal's species to face the challenging times.

\section{Species and usage with respect to Hanji}

Hanji, a community from Kashmir, is mainly living near water bodies like (Dal, Wular, Manasbal, and Jhelum) and depends on fish for livelihood. We documented seven fish species (alghad snow trout, hill trout, common carp, kunar snow trout, chirruh snow trout, rainbow trout, brown trout) among which 5 were from family Cyprinidae and 2 were Salmonidae. The Hanji community is known for fish throughout the valley (Kashmir). They are seen on the shores of water bodies, also they move from one place to another for sale of fish. While documenting we observed a strong economic bond between Hanjij community and fish. Similarly, Muhammad et al. (2017) reported the interaction between fishes and the Punjabi people Pakistan. Fish were used primarily for meat (Table 2). Locals usually consume these fish for famous recipes like Muj Gaad, Nadur Gaad. The fish waste in Kashmir is believed to increase the growth of bottle-gourd and cucumber plants. Fisheries form an important constituent of the economy of Jammu and Kashmir and contribute a significant $23 \%$ to its Gross State Domestic Product (GSDP) (https:// knskashmir.com Dec 2016; Rashid \& Singh 2020). In addition to this, fisheries are linked with agricultural activities and contribute to the economy of the state and generate self-employment (Rashid \& Singh 2020).

\section{Feed Habit}

The present study documented a variety of natural feeds (Fig. 6) in the form of, natural pastures, forest lands, paddy, paddy grass, tree leaves, maize straw, maize, aquatic weeds, community lands, phytoplankton, small insects, and zooplanktons for the commercial documented species. The present study documented only natural feeding sources as commercial feeds are expensive, and the local population believes that the use of natural herbs keeps animal species healthy and protects them from various diseases. Different studies from the Jammu and Kashmir have reported the use of medicinal herbs for the wellbeing of livestock (Dutta et al. 2021; Dar et al. 2018) For winter, when the vegetation is dormant the farmers make use of hay, dried tree leaves, etc., which are processed in autumn. The birds feed on insects like ants, earthworms, beetles, bark beetles, and grasshoppers, along with this they also take advantage of agriculture by consuming grains of paddy, maize, wheat, etc.

\section{Market value}

For any commercial species, the market value is an important parameter that rises and falls because of various factors like demand, production, inflation (Boyd et al. 2001). Our documented species' market value ranged from 200 to 1000 Indian rupees per kilogram (Fig. 7; Table 2). Normally intermediary traders buy the animals from the owner and sell them to the market and the market venders to the customers. In this small commercial chain naturally the price increases from a primary seller to the final customer (Primary owner $>$ Interconnector $>$ Market $>$ Final Customer). The maximum price per $\mathrm{kg}$ was observed in Kashmiri jungle fowl with a market value of $1000 \mathrm{Rs} / \mathrm{kg}$, interconnector value $800 \mathrm{Rs} / \mathrm{kg}$, and basic owner value $600 \mathrm{Rs} / \mathrm{kg}$. 


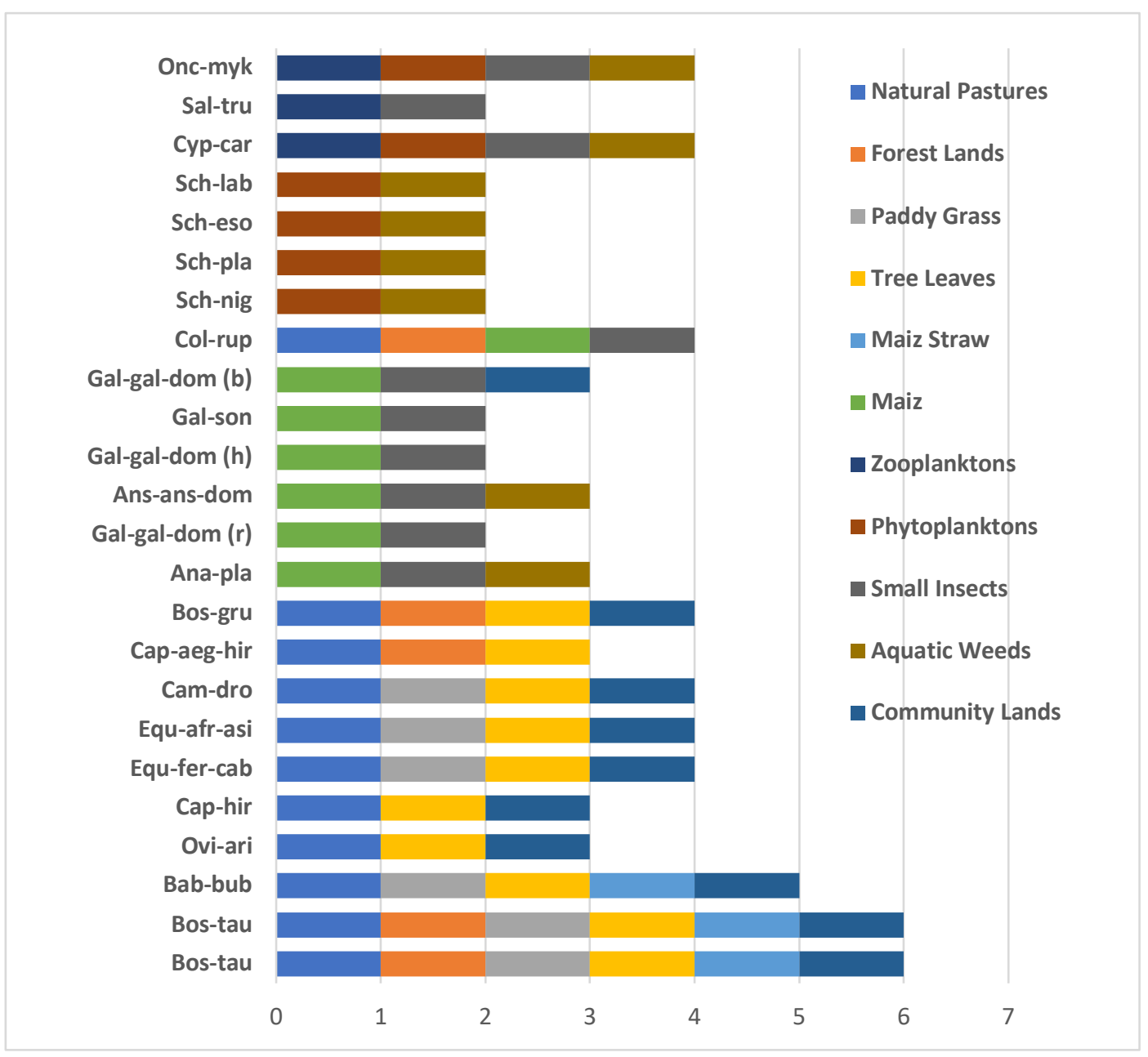

Figure 6. Feed pattern of commercial species.

The lowest price/kg was seen in Broilers with a market value $160 \mathrm{Rs} / \mathrm{kg}$, interconnector value $130 \mathrm{Rs} / \mathrm{kg}$, and basic owner value $100 \mathrm{Rs} / \mathrm{kg}$ (Fig. 7). This can be explained by the fact that Kashmiri jungle fowl is captured from the forest by the Pahari people which requires a lot of time and energy, hence is valued high. On other hand, Broilers are reared in farms for meat hence the value is low. Indigenous fish (alghad snow trout, hill trout, chirruh snow trout, kunarsnow trout) are more highly valued than exotic fish (common carp, brown trout, rainbow trout), because locally it is believed that the indigenous species have traditional medicinal uses like increasing milk production in lactating mothers, increasing virility, provide strength to the body, smoothen the skin. Raina and Petr (1999) also reported the higher value of indigenous fish than exotics. Three species (horse, donkey, and hill pigeon) were always valued with a market value of 60000 Rs, 25000 Rs, 20000 Rs, basic owner value was 57000 Rs, 23500, Rs and 18000 Rs, and the interconnector value was 58000 Rs, 24000 Rs, 19000 Rs respectively. The price of the species fluctuated. The owners often sold the species directly to the market, even on some special occasions like festivals, marriage, parties the owner directly sells the species to the consumer for meat, bypassing the market and intermediaries.

\section{Cultural and religious uses}

Many of the documented species had a strong relation with local cultural aspects and religion. In the valley (Kashmir) ox is used to plough the backyard, a ritual locally called "Goongul". This ritual is having the message that one who will work hard in fields during the season will get fruits in the end. People with Hindu faith believe that the ox is the vehicle (Vahana) of Lord Shiva, cow is treated as sacred and called "govmata" or Kamadhenu and worshiped. Cow udders symbolize four objectives (Purushartha), (i.e., dharma or righteousness, artha or material wealth, kama or desire, and moksha or salvation); her horns symbolize the gods, her face the sun and moon, and her shoulders the god of fire (agnidev). As these animals are linked with the faith, the people of the respective 
religion do not use them for commercial purposes. Muslims generally do use these animals for commercial purposes and in their religious ceremonies, like on Eid, they sacrifice animals like, cows, ox, sheep, camel, and buffalo. At the same time, Muslims use these animals in marriages, funerals, festivals, and other special occasions.

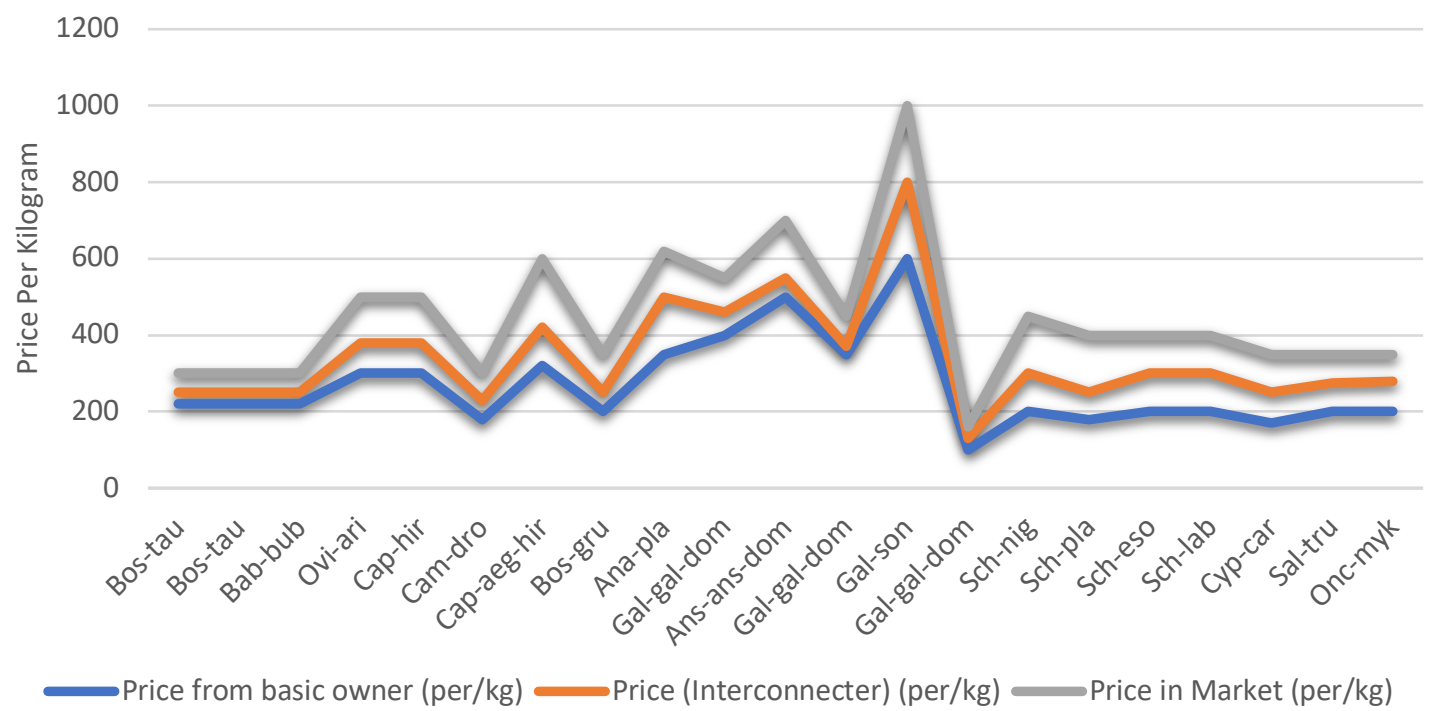

Figure 7. Market value of commercial livestock species.

The rooster is praised in Muslim culture as it crows in the early morning to announce prayers. Many Islamic scholars say that the rooster receives the signal for crowing from an angle called "Deek", which is why in Arabic the rooster is named as "Deekun". In Buddhism yak is treated as sacred animal, and it is believed that yak head, eyes intestine, hair, hoofs, and heart are transformed into sun, moon, stars, rivers, lakes, forests, and mountains, meanwhile, bones represent the symbol of purity, strength, and courage. Sikhs believe that there is a spark in every animal's soul and don't own such sacred animals. Magicians use the blood of the pigeon to perform black magic. Likewise, some fish are also used for black magic, these magicians in Kashmir are called Jooadgar, in Jammu Tantaric and in Ladakh Langtoo. Sheep is regarded as synonymous for a healthy and masculine body structure, and donkey is attributed to foolishness. Changthangi is unique to Changapa and is used to produce unique wool called Pashmina. Shawls are made from this pashmina which are having a very high price in the international market. These Shawls are so smooth that they can be made to enter through a ring easily (Wani et al. 2009). The Hanji community sundries the fish and fingerlings in summer for later usage in winters, these dried fish are called Hugaad.

\section{Potential Threats to the commercial species}

The commercial species documented from the present study do face some potential threats like diseases and predation. We focused on the predation part to document the top main predators seen locally by the livestock owners.

\section{Predation}

Predation is the main factor that causes mortality in livestock. The present study documented eight main predators (Selenarctos thibetanus, Vulpes vulpes, Herpestes auropunctatus, Canis familiaris, Felis catus, Canis lupus, Panthera uncia, and Panthera pardus) causing casualties (Table 2). These predators have been reported from various parts of Himalayan region (Ahmad et al.2020; Haq et al.2020). The heat map (Fig 8) shows the relationship between predators and prey in the study area and classified the predators into three groups that were recognized based on indicator species i.e., group $1^{\text {st }}$ with Selenarctos thibetanus. Group $2^{\text {nd }}$ with Canis lupus, Panthera uncia, and Panthera pardus. Group $3^{\text {rd }}$ with Vulpes vulpes, Herpestes auropunctatus, Canis familiaris, Felis catus.

In Ladakh Panthera uncia, Canis lupus, and Selenarctos thibetanus were found affecting the commercial species especially, as these threatened carnivores are often found close to human habitation and sometimes prey on peoples' livestock. A carnivore depredation study revealed three villages in Gya-Miru (Ladakh) lost 1.9 livestock 
heads per household per year, resulting in a monetary loss of \$12,120 (Namgail et al. 2007). In Jammu and Kashmir Panthera pardus, Selenarctos thibetanus, Vulpes vulpes, and Herpestes auropunctatus were causing depredation of commercial species. Rao et al. (2002) reported the commercial species depredation from Nanda Devi Biosphere reserve India. Globally, at least two dozen species of terrestrial carnivores' prey on commonly found domesticated animal species (Inskip and Zimmermann2009; Nyhus 2016), it for this people around the world including our selected study area have expressed deep hostility towards large carnivores. The local inhabitants while going through the loss of the animal species were found to be in the capacity to take revenge by killing the predator species resulting human wildlife conflict and ultimately posing a threat to wildlife biodiversity.

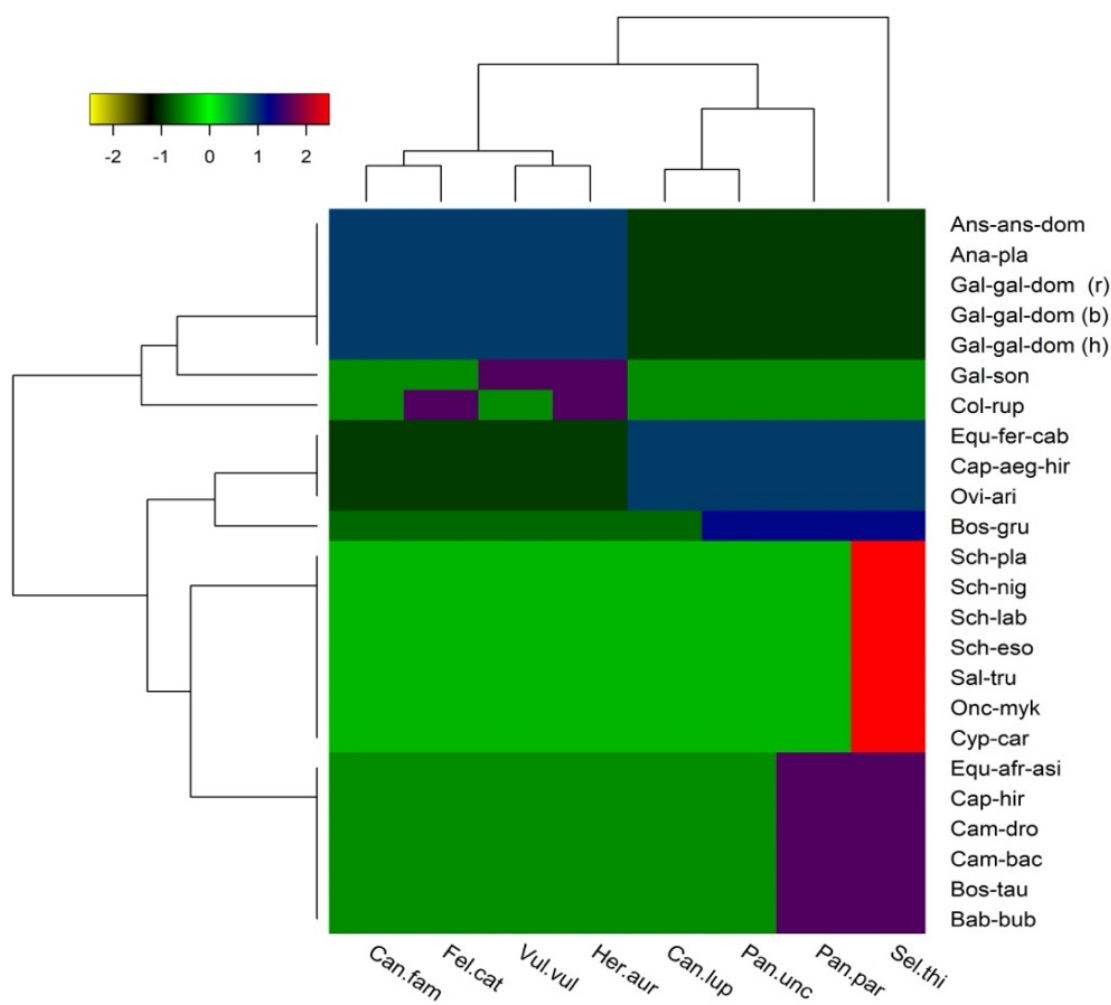

Figure 8. Two-way cluster showing relationship between predicator and commercial species.

\section{Conclusions}

The interaction between people and nature is important for the well-being of mankind. The fauna is a vital asset and has a direct implication for local livelihoods. Different species are specific to particular ethnic groups, forming an important part of their culture, economy, and agriculture, as in the present study fish were found specific to Hanji, Kashmiri jungle fowl to Pahari and Changthangi specific to Changapa, playing a vital role in the economy of these ethnic groups. Understanding the usage of commercial species in a specific ethnic group will help the stakeholders to draft better policies to overcome economic crises. Conservation mitigating measures can be put into action to overcome human wildlife conflicts due to predation which in turn would prevent the killing of wild animals and would safeguard the diversity of wild fauna in the Himalayan region. The ethnic groups of Jammu, Kashmir and Ladakh hold specific beliefs on utilization of parts of different animals, and most of the population in these regions are non-vegetarian.

\section{Declarations}

Ethics approval and consent to participate: All the participants provided prior informed consent before the interviews.

Availability of data and materials: Data is available from the first author.

Competing interest: The authors declare that they have no competing interests.

Funding: The research did not receive any specific grant from funding agencies in the public, commercial, or not -for-profit sectors 
Author contributions: $\mathrm{MH}$ carried out the field study. $\mathrm{SMH}$ and $\mathrm{MH}$ analyzed and interpreted the data and results. $\mathrm{MH}$ and SMH wrote the manuscript. UY, SH, MA, RWB revised the manuscript. All authors read and approved the final manuscript.

\section{Literature cited}

Ahmad K, Bhat BA, Ahmad R, Suhail I. 2020. Wild mammalian diversity in Jammu and Kashmir state. In Biodiversity of the Himalaya: Jammu and Kashmir State. Springer, Singapore.

Ahmad S, Mir NH, Bhat SS, Singh JP. (2018). High Altitude Pasturelands of Kashmir Himalaya: Current Status, Issues and Future Strategies in a Changing Climatic Scenario. Current Journal of Applied Science and Technology 1-10.

Ali, J. 2007. Livestock sector development and implications for rural poverty alleviation in India. Livestock Research for Rural Development 19:1-5.

Altaf A, Haq SM, Shabnum N, Jan HA. 2021. Comparative assessment of Phyto diversity in Tangmarg Forest division in Kashmir Himalaya, India. Acta Ecologica Sinica. doi: 10.1016/j.chnaes.2021.04.009

Altaf M, Javid A, Umair M, lqbal KJ, Rasheed Z, Abbasi AM. 2017. Ethnomedicinal and cultural practices of mammals and birds in the vicinity of river Chenab, Punjab-Pakistan. Journal of Ethnobiology and Ethnomedicine13:1-24.

Altaf M, Umair M, Abbasi AR, Muhammad N, Abbasi AM. 2018. Ethnomedicinal applications of animal species by the local communities of Punjab, Pakistan. Journal of Ethnobiology and Ethnomedicine 14:1-25.

Alves RR, Rosa IL, Neto NAL, Voeks R. 2012. Animals for the gods: magical and religious faunal use and trade in Brazil. Human Ecology 40:751-780.

Asif M, Haq SM, Yaqoob U, Hassan M, Jan HA. 2021. A preliminary study on the ethno-traditional medicinal plant usage in tehsil "Karnah" of District Kupwara (Jammu and Kashmir) India. Ethnobotany Research and Applications 21:1-14.

Beetz A, Uvnäs-Moberg K, Julius H, Kotrschal K. 2012. Psychosocial and psychophysiological effects of humananimal interactions: the possible role of oxytocin. Frontiers in Psychology3:234.

Bentley J. 2019. U.S. per capita availability of red meat, poultry, and seafood on the rise USDA -Economic Research Service. https://www.ers.usda.gov/amber-waves/2019/december/us-per-capita-availability-of-red-meat-poultryand-seafood-on-the-rise/

Boyd JH, Levine R, Smith BD 2001. The impact of inflation on financial sector performance. Journal of Monetary Economics 47:221-248.

Chellappandian M, Pandikumar P, Mutheeswaran S, Paulraj MG, Prabakaran S, Duraipandiyan V, Ignacimuthu S, AlDhabi NA. 2014. Documentation and quantitative analysis of local ethnozoological knowledge among traditional healers of Theni district, Tamil Nadu, India. Journal of Ethnopharmacology 154:116-130.

Cheung PCK, Mehta BM. (Eds). 2015. Handbook of food chemistry. Springer, Berlin. Heidelberg,2015.

Dar MS, Khuroo AA, Malik AH, Dar GH. 2018. Ethno-veterinary uses of some plants by Gujjar and Bakerwal community in Hirpora Wildlife Sanctuary, Kashmir Himalaya. SKUAST Journal of Research 20:181-186.

Dutta, A, Singh K, Singh B, Sharma YP. 2021. Exploring Traditional Veterinary Practices from Gujjar and Bakarwal Tribes of District Poonch, Jammu \& Kashmir: A Boon for Animals from Our Ancestors.

Fisheries and aquaculture Gov. of India 2020 (http://www.fao.org/fishery/countrysector/naso_india/en\#: : text=India\%20is\%20also\%20an\%20important,about\%209.06\%20million\%20metric\%20tonnes).

Frankl[c T, Voljč M, Salobir J, Rezar V. 2009. Use of herbs and spices and their extracts in animal nutrition. Acta Agricola Sloveniae 94:95-102.

Gairola S, Sharma,J, Bedi YS. 2014. A cross-cultural analysis of Jammu, Kashmir and Ladakh (India) medicinal plant use. Journal of Ethnopharmacology 155:925-986.

Gov. of Jammu and Kashmir 2020 (https://jk.gov.in/jammukashmir/?q =demographics) 
Haidar R. 2021. Chemical composition, traditional and modern uses of meat of animals - a review. Journal of Wildlife and Ecology 5: 47-55.

Hamad MNE, Baiomy AA. 2010. Physical properties and chemical composition of cow's and buffalo's milk in Qena governorate. Journal of Food and Dairy Sciences 1: 397-403.

Haq SM, Calixto ES, Yaqoob U, Ahmed R, Mahmoud AH, Bussmann RW, Mohammed OB, Ahmad K, Abbasi AM (2020a). Traditional Usage of Wild Fauna among the Local Inhabitants of Ladakh, Trans-Himalayan Region. Animals 10:2317.

Haq SM, Khuroo AA, Malik AH, Rashid I, Ahmad R, Hamid M, Dar GH. 2020. Forest ecosystems of Jammu and Kashmir State. In Biodiversity of the Himalaya: Jammu and Kashmir State . Springer; Singapore,2020

Haq SM, Singh B. 2020. Ethnobotany as a Science of Preserving Traditional Knowledge: Traditional Uses of Wild Medicinal Plants from District Reasi, J\&K (Northwestern Himalaya), India. In Botanical Leads for Drug Discovery. Springer, Singapore, 2020.

Hoffman JR, Falvo MJ. 2004. Protein-which is best? Journal of Sports Science \& Medicine 3:118.

HuiY H. 2012. Handbook of Meat and Meat Processing. CRC press.

Inskip C, Zimmermann A 2009. Human-felid conflict: a review of patterns and priorities worldwide. Oryx 43:18-34. Jina PS. 1996. Ladakh: The land and the people. Indus Publishing, 1996.

Keeton J T, Eddy S. 2004. Chemical Composition. Enc. Elsevier Academic Press, Oxford, 2004.

Kumar M, Kumar V, Roy D, Kushwaha R, Vaiswani S. 2014. Application of herbal feed additives in animal nutritiona review. International Journal of Livestock and Research.

Mabbett I. 1986. Buddhism in Champa. Southeast Asia in the 9th to 14th Centuries, Cambridge University Press, 1986.

Mahawar MM, Jaroli DP. 2006. Animals and their products utilized as medicines by the inhabitants surrounding the Ranthambhore National Park, India. Journal of Ethnobiology and Ethnomedicine 2: 1-5.

Mahawar MM, Jaroli DP. 2007. Traditional knowledge on zootherapeutic uses by the Saharia tribe of Rajasthan, India. Journal of Ethnobiology and Ethnomedicine 3:1-6.

Martos Martinez-Caja, A. 2021. A friend in need is a friend indeed: The benefits of the Human-Animal Bond during challenging times (Doctoral dissertation, Ghent University).

Merlin Junior IA, Sifuentes dos Santos J, Grecco Costa L, Grecco Costa R, Ludovico A, de Almeida Rego FC, Walter de SantanaEH 2015. Sheep milk: physical-chemical characteristics and microbiological quality. Archivos Latinoamericanos de Nutricion 65: 193-198.

Ministry of Agriculture Department of Animal Husbandry, Dairying and Fisheries 2012. 19th Livestock Census. 2012. All India Report. Ministry of Agriculture Department of Animal Husbandry, Dairying and Fisheries, Krishi Bhawan, New Delhi. http://dahd.nic.in/sites/default/filess/Volume\%20II.pdf

Mir NH, Ahmad SU, Verma DK. 2016. Livestock rearing-sectorial status and fodder-feed strategies in Kashmir Himalayas. Annals of Biology 32: 253-9.

Mola MG, Hailie YY, Terefe, HB, Kessete RY. 2020. Ethnozoological Study of Traditional Medicinal Animals and Their Products Used by The People of South Achefer District, Northern Ethiopia.

Mughal S, Pervaz, Bashir, Shamashad. 2020. Assessment of Diversity and Ethnopharmacological Uses of Birds in Chakar, Hattian Bala District, Azad Jammu and Kashmir -Pakistan. Journal of Wildlife and Ecology 4:35-44

Muhammad N, Khan AM, Iqbal KJ, Haider MS, Ashraf S, Ansari ZS, Chattha SA, Abbasi AR, Yaqoob M. 2017. Assessment of distribution and ethnocultural uses of the Baringo tilapia (Oreochromis niloticus) in Punjab, Pakistan. Journal of Wildlife Ecology 1:7-13. 
Namgail T, Fox JL, Bhatnagar YV. 2007. Carnivore-caused livestock mortality in Trans-Himalaya. Environmental Management 39:490-496.

National Research Council of the National Academies. 2015. Critical role of animal science research in food security and sustainability. In: Committee on Considerations for the Future of Animal Science Research Washington, DC: Natl. Acad. Pres

NDDB. Gov. of India 2020 (https://www.nddb.coop/information/stats/pop)

Nyhus PJ. 2016. Human-wildlife conflict and coexistence. Annual Review of Environment and Resources 41:143171.

Raina HS, Petr T. 1999. Coldwater fish and fisheries in the Indian Himalayas: lakes and reservoirs. Fish and fisheries at higher altitudes: Asia 64-88.

Rao KS, Maikhuri RK, Nautiyal S, Saxena KG. 2002. Crop damage and livestock depredation by wildlife: a case study from Nanda Devi Biosphere Reserve, India. Journal of Environmental Management 66: 317-327.

Rashid G,Singh. R. 2020. Present status of fish diversity and their conservational measures for sustainable fish production in the vaishav stream of Kashmir Himalaya, India. Journal of Critical Reviews 7:3654-3663.

Rauf K, Altaf M, Mumtaz B, Altaf M, Haider R, Safeer B, Farooq SI, Safdar L, Manzoor M, Yasrub S, Bashir SM 2017. Assessment of behavior, distribution, ecology and interaction study of Cinnamon Tree Sparrow (Passer rutilans) in district Bagh-Pakistan. Journal of Wildlife and Ecology 1:43-9.

Rizvi J. 2001. Trans-Himalayan caravans: Merchant princes and peasant traders in Ladakh. Oxford University Press, 2001.

Saleem R, Altaf MM, Umair., Amjad MS, Abbasi AA. 2021. Ethnopharmacological applications of the amphibians and reptiles among the people in the vicinity of Margalla Hill National Park, Islamabad, Pakistan. Journal of Wildlife and Ecology 5:13-25.

Shah TH, Ojha SN, Biradar RS, Ali S. 2003. Elevating fishers of Dal Lake in Jammu and Kashmir. Journal of the Indian fisheries Association 30: 173-182.

Shanmathy M, Gopi M, Beulah P. 2018. Contribution of Animal Husbandry to Indian Economy, Its Characteristics and Future: A Review. Asian Journal of Agricultural Extension, Economics \& Sociology 1-7.

Sharma SK. 2002. A Study on Ethnozoology of Southern Rajasthan. Ethnobotany. Jaipur: Aavishkar Publisher, 2002.

Sorensen TA. 1948. A method of establishing groups of equal amplitude in plant sociology based on similarity of species content and its application to analyses of the vegetation on Danish commons. Kongelige Danske Videnskabernes Selskab.; Biologiske Skrifter 5:1-34.

United Nations. 2019. Growing at a slower pace, world population is expected to reach 9.7 billion in 2050 and could peak at nearly 11 billion around 2100 UN Department of Economic and Social Affairs. https://www.un.org/development/desa/en/news/population/world-population-prospects-2019.

United States Census Bureau. 2020. U.S. and world population clock. https://www.census.gov/popclock/

Wani SA, Wani MH, Yusuf S. 2009. Economics of pashmina based trans-humance production system in cold arid region of Jammu and Kashmir. Indian Journal of Agricultural Economics 64: 902-2016-67299. 